\title{
AUTOMATIC CAMERA SYSTEM CALIBRATION WITH A CHESSBOARD ENABLING FULL IMAGE COVERAGE
}

\author{
Jürgen Wohlfeil*, Denis Grießbach, Ines Ernst, Dirk Baumbach, Dennis Dahlke \\ Institute of Optical Sensor Systems, German Aerospace Center, Rutherfordstr. 2, 12489 Berlin \\ (juergen.wohlfeil, denis.griessbach, ines.ernst, dirk.baumbach, dennis.dahlke)@dlr.de
}

\author{
Commission I, WG I/9
}

KEY WORDS: Camera Calibration, Chessboard, Stereo Vision, Optical Navigation, Stereo Camera, Multi-Camera

\begin{abstract}
:
Geometric camera calibration is a mandatory prerequisite for many applications in computer vision and photogrammetry. Especially when requiring an accurate camera model the effort for calibration can increase dramatically. For the calibration of the stereo-camera used for optical navigation a new chessboard based approach is presented. It is derived from different parts of existing approaches which, taken separately, are not able to meet the requirements. Moreover, the approach adds one novel main feature: It is able to detect all visible chessboard fields with the help of one or more fiducial markers simply sticked on a chessboard (AprilTags). This allows a robust detection of one or more chessboards in a scene, even from extreme perspectives. Except for the acquisition of the calibration images the presented approach enables a fully automatic calibration. Together with the parameters of the interior and relative orientation the full covariance matrix of all model parameters is calculated and provided, allowing a consistent error propagation in the whole processing chain of the imaging system. Even though the main use case for the approach is a stereo camera system it can be used for a multi-camera system with any number of cameras mounted on a rigid frame.
\end{abstract}

\section{INTRODUCTION}

\subsection{Motivation}

When deriving spatial information from images, the camera model is the key connection between the object and image coordinate space. Therefore the accuracy of spatial measurements that can be reached with a camera system is essentially depending on the accuracy of the geometric camera calibration. The required accuracy strongly depends on the particular application. This is why it should be evaluated carefully which camera calibration approach is used in order to reach the desired accuracy.

Another issue that showed up in the context of the stereo camera based Integrated Positioning System (IPS) (Grießbach et al., 2014) developed by the German aerospace center (DLR) is the time effort for such a calibration. Although only a small number of IPS devices (e.g. in Figure 1) exists the effort for calibration turned out to be unacceptably high using available approaches and implementations. E.g. the camera calibration of one system took about a full working day, mainly for image capture, formatting and clicking. Especially further research about the stability of the calibration with respect to temperature and shocks is impaired by the high calibration effort. But also the ongoing commercializing of the system as Pilot $3 D$ (Schröder and Weber, 2019) requires a faster way of calibration without loosing accuracy.

Therefore the IPS team searched for an alternative approach. It shall be based on a chessboard due to its portability and the dense points it provides. But it shall also allow a fully automatic calibration of a stereo camera system based on the images taken from the board. Moreover, it is clearly preferable to use an own implementation of the bundle adjustment that provides the full covariance matrix of the determined camera model parameters. This is important for the evaluation of the calibration results and essential to include the uncertainties of the camera model parameters in the error propagation of IPS. The lack of such a solution was one main motivation for an individual implementation.

\subsection{State of the Art}

There exists a variety of approaches for geometric camera calibration (Hieronymus, 2012). They have in common that markers or patterns are used, representing clearly visible and distinguishable object points. These object points and their corresponding image points are then used as observations to determine the parameters of the camera model(s) and the relative orientation(s). There are three main classes of markers: circular markers, collimated light pattern, and square markers representing object point locations.

Circular markers are often claimed to provide a higher accuracy (up to $1 / 50$ of a pixel (Heikkila, 2000)) but the larger they appear in the image the more important are corrections due to perspective and camera distortion (Rudakova and Monasse, 2014). The high effort needed here and the low flexibility in resolution and object distance makes them difficult to use. Automatic detection of the markers is possible, such as X-Tag (Birdal et al., 2016), for example. However, in rather natural environments there is a good chance to detect many false circular features as well. The calibration software Australis (Photometrix, 2001) uses such markers, for example.

Collimated light pattern can be created by a diffractive optical elements (DoE) (Grießbach et al., 2008) or, sequentially, with the classical collimator/goniometer approach. Both methods simulate points at infinity projected onto the focal plane with precisely known directions. Points on the image can automatically be assigned to the known directions and the centroid can be determined with high precision. This approach especially suits 
single cameras focused to infinity or close to infinity. It has its limitations for stereo camera systems as the aperture of the collimated light is too small for most stereo baselines. However, both methods are practiced in DLR and are under further development that could make them more suitable for near-field stereo camera systems in the future.

Square markers provide corners or crossings of typically rectangular patterns to represent object points with known position. The visual position of single outer or inner corners in an image varies significantly with the image exposure and with the point spread function (PSF) over the field of view (FoV). The solution is the use of chessboard patterns where the outer corners of two black and two white fields meet. Here the previously mentioned effects are neutralized due to the symmetry of the pattern. Due to the ease of use, this method became very popular in the last years. Automatic chessboard recognition is implemented in the OpenCV camera calibration (Bradski, 2010), for example. A great advantage in comparison to circular markers is that they are almost invariant to scale. The accuracy of the detected corner is in the order of $1 / 20$ to $1 / 60$ of a pixel (Abmayr et al., 2008).

With collimated light patterns a slightly higher accuracy can be achieved when using models of the PSF. However, chessboardlike markers are chosen for the calibration of stereo camera systems due to the above mentioned advantages.

Different available approaches using chessboards have been used so far. The OpenCV camera calibration (Bradski, 2010) supports automatic detection of the chessboard but it turned out to be too unreliable for poses viewing the chessboard at a steep angle. It even completely fails by design if only one corner of one of the chessboard fields is outside the image. This restriction causes a concentration of points in the center of the images and a underrepresentation of the outer ranges of the FoV as seen in Figures 5 and 6. This leads to an inaccurate distortion calibration, especially in the under-represented ranges.

The DLR Camera Calibration Toolbox (Strobl et al., 2010; Strobl and Hirzinger, 2008, 2011a) overcomes that problem with the help of three circles in the middle of the chessboard used as a starting point to search as many corners as visible in the image. The achieved calibration results meet the requirements of IPS. But it has the disadvantage that the automatic recognition of the three marker points often fails at images from steep angles and if background is visible. So in practice the three points have to be clicked manually in every image resulting in an unacceptably high workload.

Various approaches are using fiducial markers to assign the detected chessboard corners with the corresponding object point coordinates automatically, such as Aruco (Romero-Ramirez et al., 2018) or CALTag (Atcheson et al., 2010). These markers are not designed for an accurate determination of their position but they have the great advantage that they are distinguishable automatically. They are usually inserted in or next to chessboard fields helping to assign close chessboard corners, which are then used for calibration.

These markers allow an automatic point assignment even if the chessboard is not completely visible in the image. This enables the desired distribution of points as seen in Figure 6 without manual intervention. A clear disadvantage of the above mentioned solutions is that every (or every second) field of the chessboard requires a unique tag. This limits the number of fields a chessboard can have. The more fields are required the larger the codes

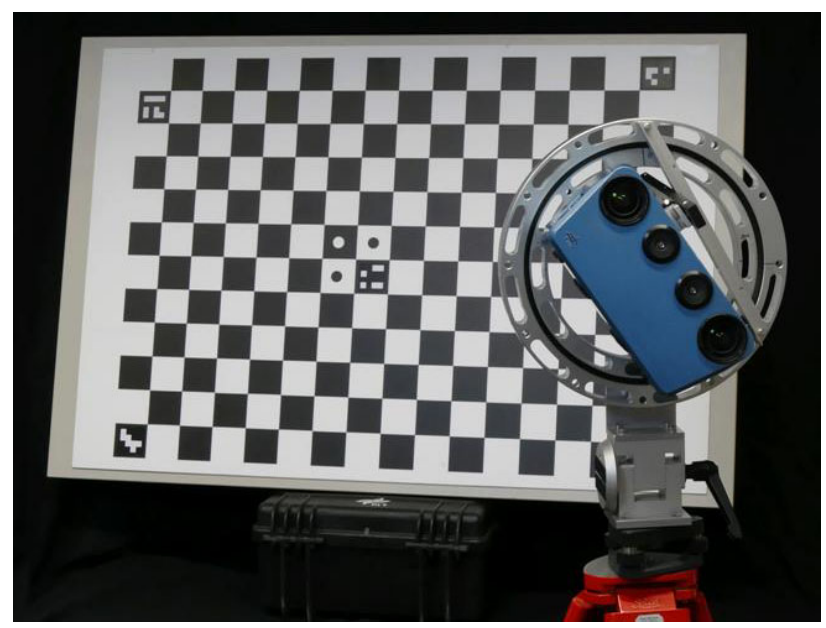

Figure 1: Background: Calibration board with AprilTags in the middle and the four corners as well as the three circles in the middle used for DLR Camera Calibration Toolbox. Foreground: DLR's Integrated Positioning System with two cameras (outer) and two LEDs (inner)

of the tags have to be and the more difficult it is to recognize and decode them from steep angles and with low resolution.

Although the use of tags for the assignment of points is regarded to be very helpful a solution was searched to overcome the problem with an approach without limitation of the number of fields. The obvious solution is using only few tags with short codes that are well distributed over the chessboard so that at least one is visible in each image. Few tags allow short codes that enable a robust automatic detection at low resolution, even in images taken from steep angles and larger distances. This approach is described in the following section.

\section{PROPOSED CALIBRATION METHOD}

\subsection{Overview}

In this paper a camera calibration approach is proposed that satisfies the following requirements:

- applicable for mono and multi-camera systems, e.g. stereo

- suitable for mobile and stationary calibration targets

- allowing a large number of well distributed calibration points

- automated point assignment, even in extreme perspectives

- high accuracy and repeatability of the calibration result

- bundle adjustment with model parameters covariance matrix

- open for the usage of multiple chessboards in one setup

The following subsections describe the five main steps of calibration:

- Choice and creation of the calibration target (Section 2.2)

- Image acquisition and preparation (Section 2.3)

- Identification of the chessboard corners in the images (Section 2.4) 
- Assignment of the object coordinates with the found corners in the images (Section 2.5)

- Calculation of the model parameters and covariance using a bundle adjustment (Section 2.6)

\begin{tabular}{lccccc}
\hline & Partial & Auto & Cov & Stereo & Multi \\
\hline OpenCV & - & + & - & $\circ$ & - \\
MATLAB $^{\circledR}$ (Bouguet) & - & - & + & $\circ$ & - \\
CalDe/CalLab (DLR) $^{\text {This approach }}$ & + & $\circ$ & + & + & - \\
\hline
\end{tabular}

Table 1: Comparison of different calibration approaches in terms of their capabilities to handle a partially visible board (Partial), no need of manual interactions (Auto), output of covariance matrix (Cov), and usability for stereo systems (Stereo) or multi camera systems (Multi). Details are found in Section 3.

\subsection{Chessboard}

Among a variety of calibration targets the chessboard (checkerboard) satisfies best the needs of a stereo camera calibration. It is much easier to produce then its alternatives. Even though it is possible to come along with a non-flat chessboard (Strobl and Hirzinger, 2011b) the presented approach assumes a flat board. This makes it much simpler to specify the point locations by just defining a flat grid interval and the number of rows and columns and avoids additional unknowns.

In order to achieve accurate calibration results the imperfections on the surface should be less than $1 \mathrm{~mm}$ for a chessboard with a size of about $1 \mathrm{~m}$. However, this value can differ depending on the application-specific accuracy requirements. The board must be stable enough to stay within this range even if it is moved or if temperature or humidity changes. Under certain circumstances a $20 \mathrm{~mm}$ coated particle board can meet these conditions but it is sensitive to changing humidity. Best experiences so far have been made with a aluminum honeycomb board.

The chessboard can be printed with a large sized printer and then glued onto the board, but its real dimensions have to be checked carefully after the print. Often individual scale factors of each dimension can be detected by measuring the distances all over the board. These scale factors can then be added to the definition of the board.

As every chessboard has certain tolerances and uncertainties it is not only important to keep these uncertainties within an acceptable range. It is also important to estimate the uncertainties as they are used as input for the error propagation, as explained in Section 2.6.

To prepare the board for automatic corner assignment it is equipped with one or more AprilTags (Olson, 2010). It has proven to be sufficient to add one tag in the middle of the board and one at each corner (see Figure 1). In order to support the recognition of the tags under difficult perspectives, the smallest tag set with 4 by 4 fields (tag16h5) was chosen. These fields are surrounded by a black frame with the width of one field. The outer border of this frame must be well contrasted to enable the algorithm to detect the tag. As the black chessboard fields already have such an outer border the advantage is taken to add AprilTags with the size of the black chessboard fields to make them as large as possible. However, our approach also allows adding AprilTags that are larger than one field at the price of loosing some chessboard corners where covering them. The positioning of the AprilTags is not crucial. So it is possible to simply stick printed AprilTags manually onto an existing board. It is just important not to cover the edges of chessboard fields slightly by the edges of AprilTags because this could change the visual position of the chessboard corners. When sticking AprilTags inside the black fields of the board it is useful to cut out the AprilTags along the middle of the black frame to avoid issues here.

The geometry of the chessboard is described by the number and the size of the chessboard fields in $x$ - and $y$-direction. In addition, the code numbers, sizes and positions of the AprilTags is given in a table. The AprilTags system was chosen because the team had experience with it. Any other comparable tag system is expected to work as well.

\subsection{Image Acquisition and Preparation}

The correct acquisition of the calibration images is crucial for the quality of the calibration result.

Camera Poses: First, the poses of the camera or stereo camera have to be chosen wisely. As far as possible, the whole image(s) shall be covered with points in order to provide enough measurements for the camera model that is intended to be valid for the whole image(s). The calibration board shall be captured from different positions with rotations around all three spatial axes. At least the eight poses described in Luhmann (2000, chapter 7.4.2) should be performed. This is essential to separate the model parameters as far as possible. The steeper the angles of view are, the better results can be expected.

Object distance: The correct choice of the distance from the board can also have an influence on the results. Remind that the camera model assumes a pinhole model for a lens optics. This model is only valid for a certain range of object distances as it neglects the dependency of the image distance from the object distance (thin lens formula). Therefore the calibration board should be imaged from a range of distances being representative for the object distances occurring in the scenario of typical usage of the system.

Saturation: Markers and patterns used for calibration are usually black and white in order to make the visibility as good as possible and to have a good signal to noise ratio. But most camera's automatic exposure controls usually overexpose small white areas, such as the white fields of the markers/patterns. The problem is that the border between black and white parts of the marker/pattern virtually shift in the image when the sensor saturates. This is because the gray pixels, located on the border of the pattern become lighter for longer exposures while the already saturated pixels remain white. Both, circular markers and chessboard corners widely compensate for this effect by neutralizing it at opposing edges. But it can easily be avoided completely if the camera exposure is reduced to a value that does not overexpose white regions.

Another important practical aspect is the image preparation. Usually it is necessary to select, convert, rename and/or number the captured images in a way that allows the calibration program to handle them. As almost every imaging system is different by means of data structure and format there is no general solution for this problem. But it is important to be considered in terms of saving time and effort and preventing human mistakes that are likely to occur at such a monotonous task. 
All these prerequisites are considered to be valid for most of the calibration methods mentioned in the introduction. Unfortunately they are not always achievable due to limitations of different methods. After taking the calibration images the next step is to find the chessboard corners in the images, as described in the following section.

\subsection{Corner Finding}

Only the corners where four chessboard fields meet are used for calibration. They are called corner points in the following.

These corner points are found in two steps: First, candidates for corner points are searched with particular attention to the accuracy of the edges' position. Second, these candidates are analyzed to be an actual corner point of the chessboard in order to reduce the number of false positives to a minimum.

For the first step the AGAST corner extractor (Mair et al., 2010) was used. It proved to work best on the saddle points at the corner points of the chessboard pattern. Although also corners at wrong places are selected (e.g. even on edges and homogeneous areas due to image noise) all true corners are included in the selection (high recall). As AGAST only selects corners with pixel accuracy the OpenCV function cv::cornerSubPix(...) was used to refine their position on sub-pixel level. This function is used in the same way in the OpenCV calibration approach. It is a crucial factor for the accuracy of the points and therefore for the accuracy of the quality of the calibration. However, from experience with the OpenCV calibration tools it is known to give satisfying results and was not further studied in this context.

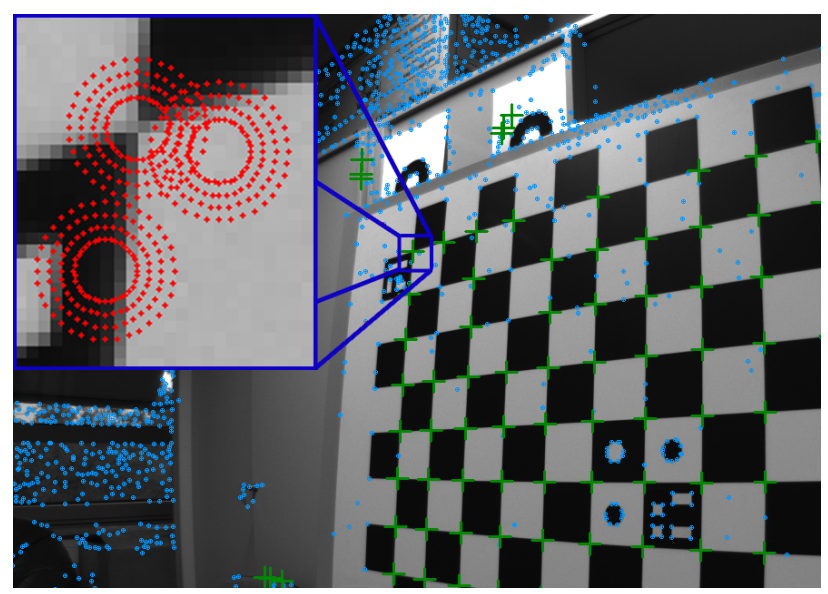

Figure 2: Selected corners by AGAST (blue dots) and verified corner points (green crosses) in an image with visible office background. Top-left: Grey value sample locations for the verification at the example of three AGAST corners.

As the second step the corner candidates are verified to be an actual chessboard corner point by the following test. In four circles closely around the position of the corner candidate (2.5 to 6.5 pixels radius) 36 gray-values are sampled at sub-pixel precision (using bi-linear interpolation), as visualized in Figure 2 (top left). Next, the mean gray-value of each circle is calculated and the indices of the samples where the gray value crosses this mean value are determined (with a small threshold in the order of the expected image noise). If the candidate lies on a chessboard corner four of such crossings are expected. Two times the gray-values cross the mean value towards lighter gray-values (rising) and two times towards darker values (falling). Moreover, the two rising and the two falling crossings are expected to be located almost exactly at the opposite side of the circle. Only corner candidates that meet the correct number and location of mean value crossings at three or four circles are considered to be corner points. There is a chance that a few false positive detections remain, mostly at structures similar to chessboard corners in the background of the scene. In practice it showed that they can be easily sorted out by the following step.

\subsection{Corner Assignment}

The corner points found in the image have to be assigned with their coordinate on the chessboard.

The coordinates of all corner points of the chessboard can be described with only four parameters: The size of the chessboard fields $\left(i_{x}\right.$ and $\left.i_{y}\right)$ and their number $\left(n_{x}\right.$ and $\left.y_{y}\right)$ in $\mathrm{x}$ and $\mathrm{y}$ direction ( $i_{x}$ and $i_{y}$ are usually identical but can differ slightly at inaccurate prints).

As all corner points of the chessboard look the same and as this approach shall not rely on the visibility of all corner points AprilTags (Olson, 2010) are used to connect the coordinate system of the chessboard with the image coordinates. The AprilTags library provides the image coordinates of the four corners of each tag in a defined order. By knowing the tag's dimension in object space, a $3 \times 3$ homography matrix can be calculated that allows the transformation of coordinates between image and board. This transformation is not precise enough to provide a usable input for the calibration. But it allows good estimates of the corner points' positions on the image in the close surrounding of the corresponding AprilTag. This helps to reliably find and assign some first corner points around the AprilTag(s). More corner points can be found by not only using the AprilTag's corners but also the already assigned corners in a distance up to 3 fields. This way the search and assignment can be continued all over the chessboard. An AprilTag is only the starting point of the search. The pseudo-code shown in Algorithm 1 describes the assignment of the corners:

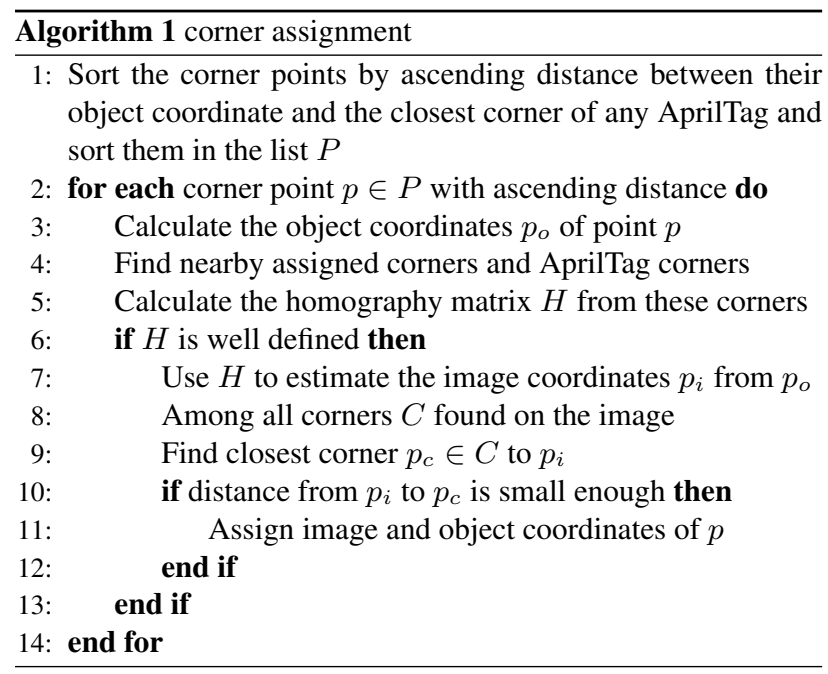

An example for the calculation of the homography is shown in Figure 3(a). The final result is displayed in Figure 3(b). It is notable that not all corner points have been assigned, even though they are visible. In order to avoid false assignments it is accepted to loose some insecure assignments. The remaining number of assigned corners is usually very high.

After all corner points in all images taken for the calibration have 


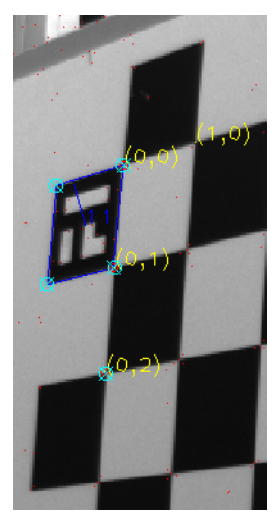

(a)

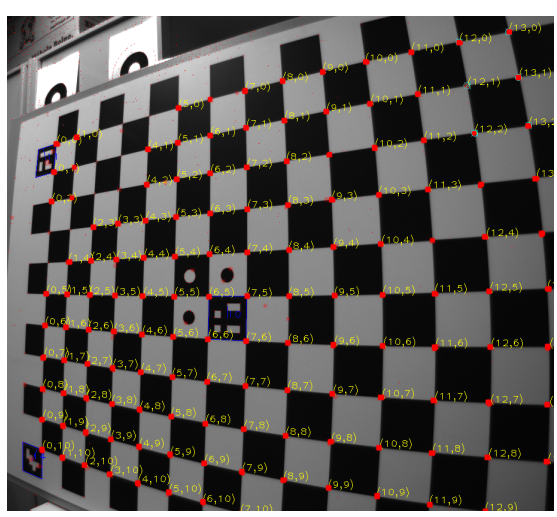

(b)
Figure 3: (a) Estimation of the image coordinate of point (1, 0 ) using the corners of the AprilTag and three already assigned points (light blue circles). (b) Result of the corner assignment

been found and assigned automatically the calibration can be performed using a bundle adjustment, as described in the following.

\subsection{Bundle Adjustment}

In order to calibrate a camera system, a geometric camera model including a distortion model needs to be defined. Both models are given in the camera reference frame $(c)$. It is aligned to rows and columns of the CCD with its origin being the intersection of the optical axis with the CCD. The world reference frame $(w)$ is defined by the chessboard itself. It is considered to be stationary by definition, even though it may be moved in the real world.

Camera Model A chessboard corner position $(X, Y, 0)^{T}$ is transformed to the camera reference frame with

$$
\left(\begin{array}{c}
X \\
Y \\
Z
\end{array}\right)^{c}=\mathbf{R}_{w}^{c}\left(\begin{array}{l}
X \\
Y \\
0
\end{array}\right)^{w}+\mathbf{t}_{w}^{c}
$$

where $\mathbf{R}_{w}^{c}$ is an orthogonal rotation matrix and $\mathbf{t}_{w}^{c}$ is the translation transforming from world to camera coordinates.

After projection to a virtual plane $\pi$ at $Z^{c}=1$, the normalized camera coordinate is given with

$$
\left(\begin{array}{l}
x \\
y
\end{array}\right)=\left(\begin{array}{l}
X / Z \\
Y / Z
\end{array}\right)^{c}
$$

The camera model allows to transfer coordinates from the camera reference frame to the individual image reference frame and vice versa. Each pixel coordinate $(u, v)^{T}$ is given in its image reference frame and can be calculated from the normalized camera coordinate $(x, y)^{T}$ with

$$
\left(\begin{array}{l}
u \\
v \\
1
\end{array}\right)=\mathbf{K}\left(\begin{array}{l}
x \\
y \\
1
\end{array}\right)
$$

where the camera matrix $\mathbf{K}$ represents a simple pinhole model, containing the principle point $\left(u_{0}, v_{0}\right)^{T}$ and the focal length $f$, which in this context, is the distance of the pinhole to the image plane.

$$
\mathbf{K}=\left[\begin{array}{ccc}
f & 0 & u_{0} \\
0 & f & v_{0} \\
0 & 0 & 1
\end{array}\right]
$$

Distortion Model The pinhole model does not consider lens distortion. It is therefore extended by the Brown-Conrady model (Brown, 1971), which consists of a radial-symmetric component $\boldsymbol{\delta}_{r}$, considering pincushion/barrel distortion and a tangential component $\boldsymbol{\delta}_{t}$. Normalized camera coordinates are distorted as follows.

$$
\left(\begin{array}{l}
x_{d} \\
y_{d}
\end{array}\right)=\left(\begin{array}{l}
x \\
y
\end{array}\right)+\boldsymbol{\delta}_{r}(x, y, \mathbf{k})+\boldsymbol{\delta}_{t}(x, y, \mathbf{p})
$$

With the radial distance $r^{2}=x^{2}+y^{2}$, the radial-symmetric model is expressed as

$$
\boldsymbol{\delta}_{r}(x, y, \mathbf{k})=\left(\begin{array}{l}
x \\
y
\end{array}\right)\left(k_{1} r^{2}+k_{2} r^{4}+k_{3} r^{6}+\cdots\right) .
$$

Although, usually there is no tangential distortion, it may occur due to manufacturing tolerances and alignment errors, e.g. decentered, shifted lenses. First introduced by Conrady and adopted by Brown (Brown, 1971), tangential distortion, also known as decentering distortion, is modeled with

$\boldsymbol{\delta}_{t}(x, y, \mathbf{p})=\left(\begin{array}{c}p_{1}\left(3 x^{2}+y^{2}\right)+2 p_{2} x y \\ p_{2}\left(x^{2}+3 y^{2}\right)+2 p_{1} x y\end{array}\right)\left(1+p_{3} r^{2}+p_{4} r^{4} \cdots\right)$.

For low order terms, the thin prism model is equivalent to this model. For higher order terms it should not be used but abandoned in favor of the tangential model (Brown, 1966).

Bundle Adjustment By using eqs. (3) to (5), the mapping from normalized camera coordinates to measured distorted image coordinates $(u, v)^{T}$ is subsumed to

$$
\left(\begin{array}{l}
u \\
v
\end{array}\right)=\left(\begin{array}{l}
u_{0} \\
v_{0}
\end{array}\right)+f\left(\begin{array}{l}
x \\
y
\end{array}\right)\left(1+\boldsymbol{\delta}_{r}+\boldsymbol{\delta}_{t}\right) .
$$

Given a set of corner points $\left(X^{w}, Y^{w}\right)^{T}$ and their corresponding measured image points $(\hat{u}, \hat{v})^{T}$ for one pose, we seek to minimize the non-linear cost function

$$
\min _{m}\left\|\left(\begin{array}{c}
\hat{u}-u_{0} \\
\hat{v}-v_{0}
\end{array}\right)-f\left(\begin{array}{l}
x \\
y
\end{array}\right)\left(1+\boldsymbol{\delta}_{r}+\boldsymbol{\delta}_{t}\right)\right\|^{2},
$$

where $(x, y)^{T}$ are calculated according to eqs. (1) and (2). The parameters $m=\left(f, u_{0}, v_{0}, \omega, \varphi, \kappa, \mathbf{t}_{w}^{c}, \mathbf{k}, \mathbf{p}\right)^{T}$ describe the interior orientation $f, u_{0}, v_{0}$, the pose with $\mathbf{R}(\omega, \varphi, \kappa)_{w}^{c}$ and $\mathbf{t}_{w}^{c}$, as well as the coefficients of the distortion model $\mathbf{k}, \mathbf{p}$. Which and how many distortion parameters need to be estimated is dependent on the used lens and has to be decided manually. The nonlinear optimization problem is solved with the Gauss-Newton algorithm utilizing the Jacobian matrix which consists of the partial derivatives of eq. (9) w.r.t. the parameters $m$.

Each additional pose contributes to the estimation of the camera model but also adds 6 parameters to the optimization problem. For a stereo system, a second camera is rigidly mounted w.r.t. the first camera which is modeled by an additional relative orientation $(\mathbf{R}, \mathbf{t})_{c 1}^{c 2}$. This approach can be easily extended in case of a multi-camera system (Choinowski et al. (2019) describes the application of this approach for a tri-focal camera system). The visualization of a Jacobian in fig. 4 nicely shows the contributions of each pose to the parameters estimation.

The cost function is minimized in a two step approach. First, by calculating the homography for each pose, the initial values for the exterior orientations as well as the interior orientation are 


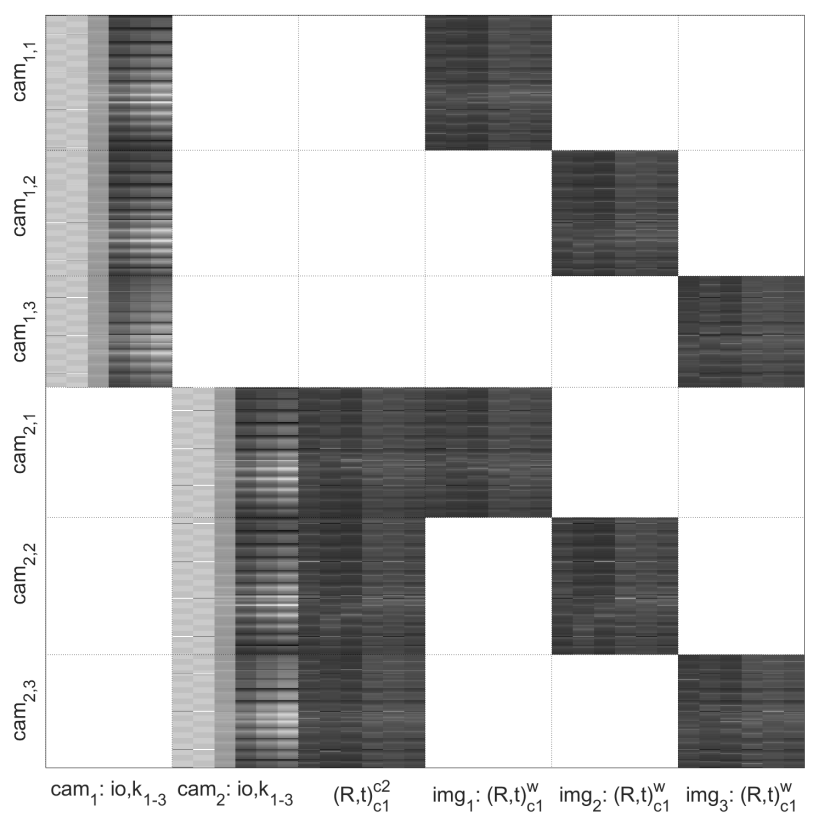

Figure 4: Jacobi matrix example for a stereo camera calibration with 3 poses. Matrix entries are scaled for better visibility.

estimated with a linear optimization (Zhang, 2000). For this step the non-linear lens distortion has been ignored. Second, these initial values are now used for the Gauss-Newton algorithm to solve the non-linear least square problem. Possible outliers, e.g. miss-matched corners, need to be detected and filtered within the optimization process. Furthermore, uncertainties in the model as well as the measured points are propagated to a full covariance matrix of the estimated parameters.

As described before the stationary chessboard defines the world reference frame. Alternatively, for a stationary camera, the camera reference frame can be used as world reference frame. This allows the user to move the chessboard itself in front of the stationary camera.

\section{PRACTICAL ASPECTS AND RESULTS}

With focus on manageability and transferability the presented camera calibration approach combines different advantages of currently available and commonly used approaches. In particular the OpenCV calibration tools (Bradski, 2010), the camera calibration toolbox implemented in MATLAB ${ }^{\circledR}$ by Bouguet (2015) and DLR Camera Calibration Toolbox (Strobl et al., 2010; Strobl and Hirzinger, 2008, 2011a), which is implemented in IDL ${ }^{\circledR}$, are used for the following comparison (see Table 1).

As already stated in section 1.2 , the capability to handle partially visible chessboards is a crucial feature. This becomes an issue when calibrating the interior orientation of a camera, using a standard calibration setup (Luhmann, 2000) and capturing the chessboard from five distinct positions at four rotation angles $\left(0^{\circ}, 90^{\circ}, 180^{\circ}\right.$ and $\left.270^{\circ}\right)$. Bradski (2010) relies on a fully seen chessboard to automate the task of point detection, while Bouguet (2015) needs the outermost corners to be clicked manually. Restricting the calibration task to completely seen chessboards, inherently leads to sparse or even no points in the outer ranges of the FoV (see Figure 5). Given this concentration of points towards the FoV's center, the estimation of radial distortion parameters consequently becomes vague for the outer FoV.
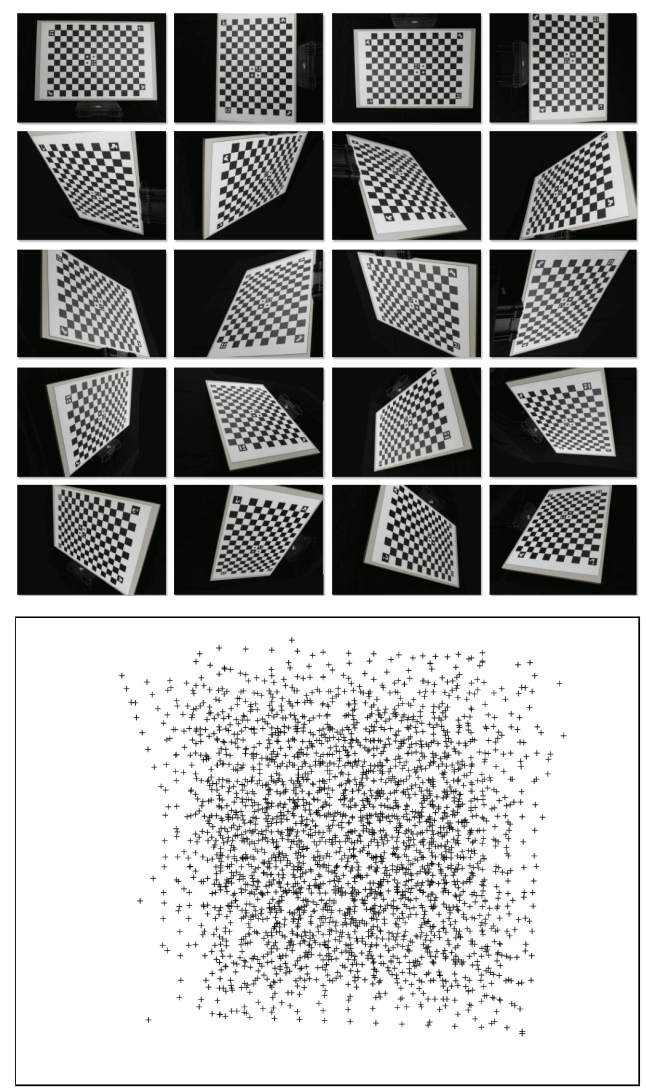

Figure 5: Typical accumulated corner distribution (bottom) for poses with the restriction of a fully visible chessboard in each image (top)

For a stereo camera setup this restriction becomes even more pronounced since it is extended to a completely visible chessboard in both images. Practically this leads to greater distances when capturing the board, which in turn means the size of the board in the image becomes smaller. The smaller the target is seen in the image, the more parallactic the angle between known points becomes, which leads to less accurate estimations of the exterior orientation.

For a stereo image pair capturing a $1 \mathrm{~m} \times 1.5 \mathrm{~m}$ chessboard with $5 \mathrm{~mm}$ focal length and $20 \mathrm{~cm}$ stereo base length, the recording distance has to be increased to $2 \mathrm{~m}$ compared to $1.3 \mathrm{~m}$ for single camera calibration. Another side effect of this restrictive setup is a shift of tie points towards one end of each camera sensor. Strobl et al. (2010); Strobl and Hirzinger (2008, 2011a) as well as the presented approach overcome this restriction and allow the usage of partially captured chessboards as long as one tag is visible (Figure 6). Hereby an equal distribution of points can be guaranteed for monocular and stereo camera calibration.

In terms of automation Bradski (2010) is comparable to the robustness of the presented approach when dealing with fully captured chessboards. Bouguet (2015) additionally requires manually and accurately clicking on the four extreme corners of the chessboard with a constant ordering rule in each image. For a stereo camera setup it becomes even more demanding since eight instead of four points have to be clicked for each image pair. When using the previously mentioned 20 standard poses and a stereo camera setup, this becomes a challenging task which is also prone to user errors. Strobl et al. (2010); Strobl and Hirzinger (2008, 2011a) uses one tag on smaller and two tags on larger 

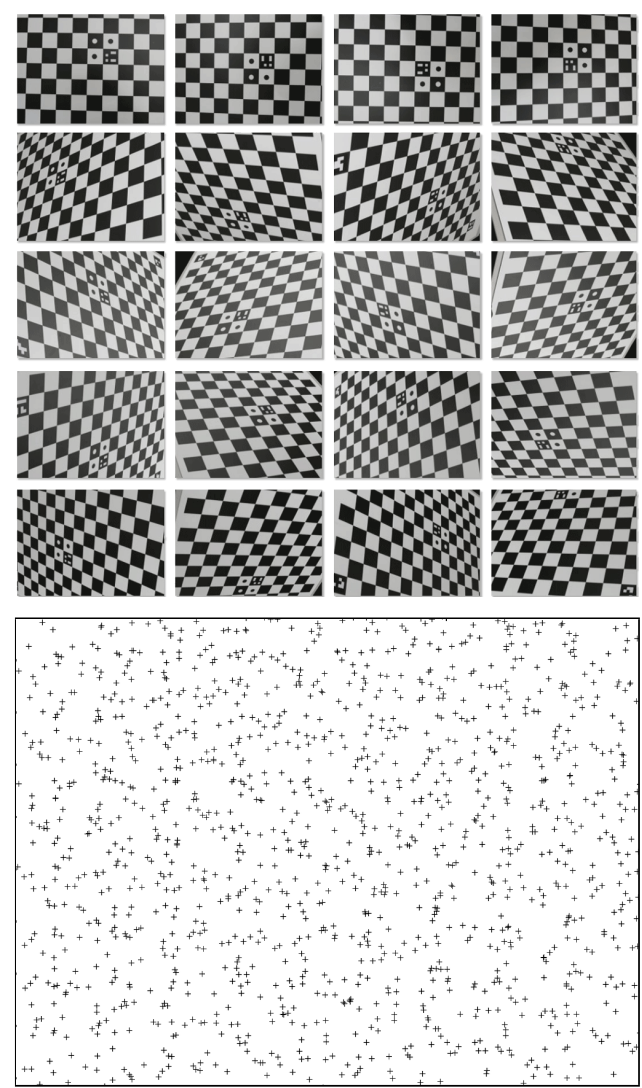

Figure 6: Well distributes corners over the whole image (bottom) due to partially visible chessboard in each image (top)

chessboards in order to automate the detection of chessboard corners. These tags are defined as either black or white filled circles.

By definition the detection of those blobs tends to find false positive especially if the chessboard environment is heterogeneous. Practically this leads to high frequent usage of the semi automatic detection setting, where three or six of these circles have to be clicked manually in each image. For the presented approach every chessboard with distinguishable AprilTags is unique and automatically evaluated even if the tags are perspectively distorted. One AprilTag in the center is sufficient for most use cases. Nevertheless four additional AprilTags can be used as backup either in the extreme corners or even nearby the chessboard.

Since calibration is often just the beginning of a processing chain, error propagation is an elementary feature. The output of covariance matrices is available for all compared approaches but Bradski (2010).

Regarding the stereo capabilities, all approaches perform the global optimization by adjusting interior orientation and relative orientation at once. An option to fix the interior orientation while performing the global bundle adjustment is also part of all approaches. In case of Bradski (2010) and Bouguet (2015) the underlying point lists have to be identical for each image pair. As a consequence both approaches fail if single fields can't be detected in one image of the pair. Strobl et al. (2010); Strobl and Hirzinger $(2008,2011 \mathrm{a})$ and the presented approach are fully compatible with different amounts of points in each image. Although the latter one has the option to set a minimum threshold for detected points. Below this threshold the corresponding image is discarded.

\section{CONCLUSIONS AND OUTLOOK}

Several tests have shown that the presented approach satisfies the requirements stated in Section 2.1. The time for automatic processing highly depends on the number of images but is convenient on a standard desktop PC. The main gain of the approach to reduce the manual work for a calibration to a minimum has been achieved. Only the capture of the images and a quick check of the results remain as human workload.

At the same time the required uncertainty information is completely available. For the calibration of the IPS system it turned out to be a very suitable solution and it is expected to be suitable for other camera systems as well. In addition the approach seems to be useful for both, stationary setups and in-field calibration.

The uncertainty calculation of the approach currently uses an assumed, uniform uncertainty of the corner positions. This can be improved using a correlation based target finder, e.g. Abmayr et al. (2008), to calculate the uncertainties of corner detection.

Basically the approach is not limited to one single chessboard. Especially for calibrations over a wider range of object distances it is planned to equip a room corner with three chessboards. Every chessboard is then equipped with distinguishable AprilTags. However, the relative orientation of the chessboards needs to be measured, for example using a tachymeter. This will be the subject of future studies.

\section{ACKNOWLEDGEMENTS}

The authors thank Michael Nitz, Karsten Stebner and Matthias Geßner (DLR Institute of Optical Sensor Systems) for providing the camera mounting setup. This work was partly funded by the EIT Raw Materials Project "UNDROMEDA".

\section{References}

Abmayr, T., Haertl, F., Hirzinger, G., Burschka, D. and Froehlich, C., 2008. A correlation based target finder for terrestrial laser scanning. Journal of Applied Geodesy 2, pp. 131-137.

Atcheson, B., Heide, F. and Heidrich, W., 2010. CALTag: High Precision Fiducial Markers for Camera Calibration. In: R. Koch, A. Kolb and C. Rezk-Salama (eds), Vision, Modelling, and Visualization (2010), The Eurographics Association.

Birdal, T., Dobryden, I. and Ilic, S., 2016. X-Tag: A Fiducial Tag for Flexible and Accurate Bundle Adjustment. 2016 Fourth International Conference on 3D Vision (3DV) pp. 556-564.

Bouguet, J. Y., 2015. Camera Calibration Toolbox for Matlab. http://www.vision.caltech.edu/bouguetj/ calib_doc/.

Bradski, G., 2010. The OpenCV Library. https: //docs.opencv.org/2.4/modules/calib3d/doc/ camera_calibration_and_3d_reconstruction.html.

Brown, D. C., 1966. Decentering distortion of lenses. Photogrammetric Engineering 32(3), pp. 444-462.

Brown, D. C., 1971. Close-range camera calibration. Photogrammetric Engineering 37(8), pp. 855-866. 
Choinowski, A., Dahlke, D., Ernst, I., Pless, S. and Rettig, I., 2019. Automatic calibration and co-registration for a stereo camera system and a thermal imaging sensor using a chessboard. In: The International Archives of the Photogrammetry, Remote Sensing and Spatial Information Sciences, Vol. in Print.

Grießbach, D., Bauer, M., Hermerschmidt, A., Krüger, S., Scheele, M. and Schischmanow, A., 2008. Geometrical camera calibration with diffractive optical elements. Optics Express 16(25), pp. 20241-20248.

Grießbach, D., Baumbach, D. and Zuev, S., 2014. Stereovisionaided inertial navigation for unknown indoor and outdoor environments. Proc. Int. Conf. Indoor Positioning Indoor Navigation.

Heikkila, J., 2000. Geometric camera calibration using circular control points. IEEE Transactions on Pattern Analysis and Machine Intelligence 22/10, pp. 1066-1077.

Hieronymus, J., 2012. Comparison of methods for geometric camera calibration. International Archives of the Photogrammetry, Remote Sensing and Spatial Information Scien me XXXIX, pp. 595-599.

Luhmann, T., 2000. Erweiterte Verfahren zur geometrischen Kamerakalibrierung in der Nahbereichsphotogrammetrie. Verlag der Bayerischen Akademie der Wissenschaften in Kommission beim Verlag C. H. Beck, Munich.

Mair, E., Hager, G. D., Burschka, D., Suppa, M. and Hirzinger, G., 2010. Adaptive and Generic Corner Detection Based on the Accelerated Segment Test. In: Proceedings of the European Conference on Computer Vision (ECCV'10).

Olson, E., 2010. AprilTag: A robust and flexible multi-purpose fiducial system. Technical report, University of Michigan APRIL Laboratory.

Photometrix, 2001. Photometrix Australis. https://www . photometrix.com.au/australis/.

Romero-Ramirez, F. J., Muñoz Salinas, R. and Medina-Carnicer, R., 2018. Speeded up detection of squared fiducial markers. Image and Vision Computing 76, pp. 38-47.

Rudakova, V. and Monasse, P., 2014. Camera Matrix Calibration Using Circular Control Points and Separate Correction of the Geometric Distortion Field. 2014 Canadian Conference on Computer and Robot Vision (CRV) pp. 195-202.

Schröder, D. and Weber, M., 2019. Precise positioning and 3d documenting of the underground workings using the dmt pilot $3 \mathrm{~d}$ navigation system. In: Occupational Safety in Industry (Russian), Vol. 4/2018, pp. 66-73.

Strobl, K. H. and Hirzinger, G., 2008. More Accurate Camera and Hand-Eye Calibrations with Unknown Grid Pattern Dimensions. In: Proceedings of the IEEE International Conference on Robotics and Automation, Pasadena, CA, USA, pp. 13981405.

Strobl, K. H. and Hirzinger, G., 2011a. More Accurate Pinhole Camera Calibration with Imperfect Planar Target. In: Proceedings of the IEEE International Conference on Computer Vision (ICCV), 1st IEEE Workshop on Challenges and Opportunities in Robot Perception, Barcelona, Spain, pp. 1068-1075.

Strobl, K. H. and Hirzinger, G., 2011b. More Accurate Pinhole Camera Calibration with Imperfect Planar Target. In: IEEE International Conference on Computer Vision (ICCV 2011), 1st IEEE Workshop on Challenges and Opportunities in Robot Perception.
Strobl, K. H., Sepp, W., Fuchs, S., Paredes, C., Smisek, M. and Arbter, K., 2010. DLR CalDe and DLR CalLab. http:// www.robotic.dlr.de/callab/.

Zhang, Z., 2000. A flexible new technique for camera calibration. IEEE Transactions on Pattern Analysis and Machine Intelligence 22(11), pp. 1330-1334. 